CRYSTALLOGRAPHIC COMMUNICATIONS

ISSN 2056-9890

Received 8 January 2016

Accepted 10 February 2016

Edited by J. Simpson, University of Otago, New Zealand

Keywords: crystal structure; imidazole derivative; new synthesis; 2-(4-methoxyphenyl)-2oxoacetaldehyde; glycine methyl ester hydrochloride.

CCDC reference: 1452746

Supporting information: this article has supporting information at journals.iucr.org/e

\section{Crystal structure of ethyl 2-[2-(4-methylbenzoyl)-5- $p$-tolyl-1H-imidazol-1-yl]acetate}

\author{
E. Arockia Jeya Yasmi Prabha, ${ }^{a}$ S. Suresh Kumar, ${ }^{a}$ Anil K. Padala, ${ }^{b}$ Qazi Naveed \\ Ahmed $^{\text {b }}$ and S. Athimoolam ${ }^{\mathrm{a} *}$
}

a Department of Physics, University College of Engineering Nagercoil, Anna University, Nagercoil 629 004, India, and ${ }^{\mathbf{b}}$ Medicinal Chemistry Division, Indian Institute of Integrative Medicine (IIIM) and Academy of Scientific and Innovative Research (AcSIR-IIIM), Jammu 180 001, India. *Correspondence e-mail: athi81s@yahoo.co.in

In the title compound, $\mathrm{C}_{22} \mathrm{H}_{22} \mathrm{~N}_{2} \mathrm{O}_{3}$, the plane of the five-membered ring is oriented at dihedral angles of $45.4(1)$ and $52.5(1)^{\circ}$ to the phenyl rings. Furthermore, this ring makes an angle of $85.2(2)^{\circ}$ with the plane of the ethyl acetate substituent. The molecular structure is affected by an intramolecular $\mathrm{C}-\mathrm{H} \cdots \mathrm{O}$ hydrogen bond between an $\mathrm{H}$ atom from the $p$-tolyl group and the carbonyl $\mathrm{O}$ atom of the acetate. The methyl group of the ethyl acetate residue is disordered over two sites with equal occupancies. The crystal structure features intermolecular $\mathrm{C}-\mathrm{H} \cdots \mathrm{O}$ and $\mathrm{C}-\mathrm{H} \cdots \mathrm{N}$ interactions. One of the $\mathrm{C}-\mathrm{H} \cdots \mathrm{O}$ hydrogen bonds forms a $C(5)$ chain motif extending along the $a$ axis. In addition, $\mathrm{C}-\mathrm{H} \cdots \mathrm{N}$ contacts form inversion dimers with $R_{2}^{2}(12)$ ring motifs, linking the imidazole ring system to the benzene ring of the $p$-tolyl substituent.

\section{Chemical context}

Imidazole and its derivatives have numerous pharmaceutical applications including uses as antifungal (Shingalapur et al. 2009), antimicrobial (Sharma et al. 2009), anti-inflammatory (Puratchikody et al. 2007), analgesic (Achar et al. 2010), antitubercular (Pandey et al. 2009), antidepressant (Hadizadeh et al. 2008), antileishmanial (Bhandari et al. 2009) and anticancer agents (Ozkay et al. 2010). We are interested in the synthesis of active pharmaceutical ingredients (APIs) based on imidazoles and we report here the synthesis and crystal structure of the title imidazole derivative.<smiles>CCOC(=O)Cn1c(-c2ccc(C)cc2)cnc1C(=O)c1ccc(C)cc1</smiles>

\section{Structural commentary}

The molecular structure of the title compound is shown in Fig. 1. The $\mathrm{C}-\mathrm{N}$ bond lengths within the imidazole ring are

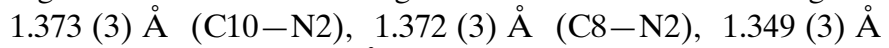
$(\mathrm{C} 9-\mathrm{N} 1)$ and $1.329(3) \AA(\mathrm{C} 10-\mathrm{N} 1)$. These bond distances 


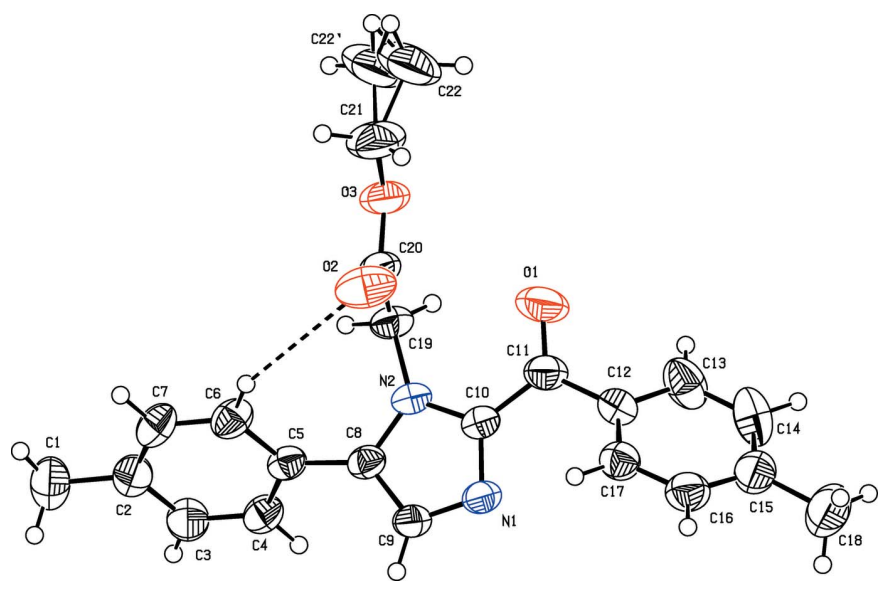

Figure 1

The molecular structure of the title compound, showing the atomnumbering scheme and $50 \%$ probability displacement ellipsoids. The methyl group (C22) of the side chain is disordered over two positions each with 0.5 occupancy.

are shorter than the single-bond length $(1.443 \AA)$ and longer than the accepted double-bond length $(1.269 \AA)$ due to electron delocalization in the central imidazole ring. The phenyl rings and the plane of the imidazole ring are inclined at angles of $45.4(1)^{\circ}$ (with the $\mathrm{C} 12-\mathrm{C} 17$ ring) and $52.5(1)^{\circ}$ (with the $\mathrm{C} 2-\mathrm{C} 7$ ring). The phenyl rings are oriented to each other with a dihedral angle of $88.1(1)^{\circ}$. Further, the imidazole ring is inclined at an angle of $85.2(2)^{\circ}$ to the best-fit plane through atoms $\mathrm{C} 19, \mathrm{C} 20, \mathrm{O} 3, \mathrm{C} 21$ and $\mathrm{C} 22$ of the ethyl acetate substituent. The molecular structure is also influenced by the formation of an intramolecular $\mathrm{C} 6-\mathrm{H} 6 \cdots \mathrm{O} 2$ hydrogen bond, Table 1, which generates an $S(8)$ ring motif (Bernstein et al., 1995).

\section{Supramolecular features}

The N-bound methylene group of the side chain is connected with the carbonyl oxygen of an adjacent molecule through a $\mathrm{C} 19-\mathrm{H} 19 A \cdots \mathrm{O} 2$ hydrogen bond, forming a linear $C(5)$ chain motif along the $a$ axis, Table 1 and Fig. 2. The phenyl and

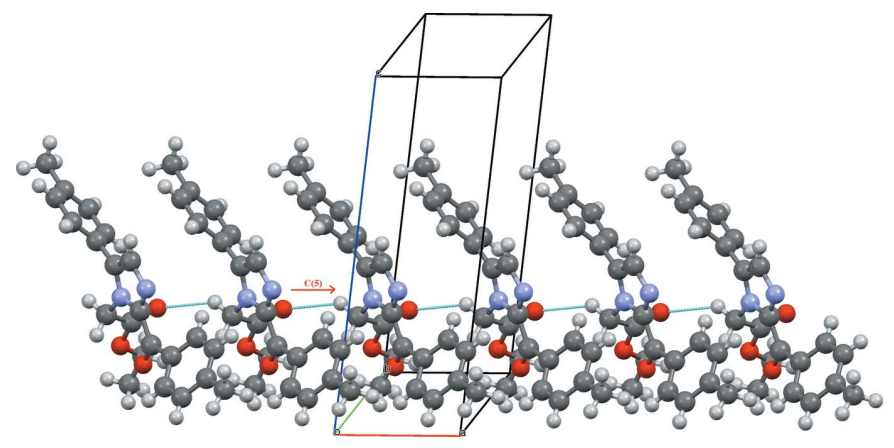

Figure 2

Linear $C(5)$ chains formed by a $\mathrm{C}-\mathrm{H} \cdots \mathrm{O}$ intermolecular interaction extending along the $a$ axis of the unit cell.
Table 1

Hydrogen-bond geometry $\left(\AA{ }^{\circ}\right)$.

\begin{tabular}{lllll}
\hline$D-\mathrm{H} \cdots A$ & $D-\mathrm{H}$ & $\mathrm{H} \cdots A$ & $D \cdots A$ & $D-\mathrm{H} \cdots A$ \\
\hline $\mathrm{C} 6-\mathrm{H} 6 \cdots \mathrm{O} 2$ & 0.93 & 2.91 & $3.723(4)$ & 147 \\
$\mathrm{C} 1-\mathrm{H} 1 A \cdots \mathrm{O} 2^{\mathrm{i}}$ & 0.96 & 2.71 & $3.605(4)$ & 155 \\
$\mathrm{C} 4-\mathrm{H} 4 \cdots \mathrm{N} 1^{\mathrm{ii}}$ & 0.93 & 2.83 & $3.724(3)$ & 161 \\
$\mathrm{C} 19-\mathrm{H} 19 A \cdots \mathrm{O} 2^{\mathrm{iii}}$ & 0.97 & 2.51 & $3.309(3)$ & 140 \\
\hline
\end{tabular}

Symmetry codes: (i) $-x,-y+1,-z+1$; (ii) $-x,-y,-z+1$; (iii) $x-1, y, z$.

imidazole rings are linked through inversion-dimer formation involving $\mathrm{C} 4-\mathrm{H} 4 \cdots \mathrm{N} 1$ hydrogen bonds that generate $R_{2}^{2}(12)$ ring motifs. A second inversion dimer to an adjacent molecule results from $\mathrm{C} 1-\mathrm{H} 1 \cdots \mathrm{O} 2$ contacts, forming ring $R_{2}^{2}(22)$ [OK?] rings, Fig. 3.

\section{Database survey}

The Cambridge Structural Database (Groom \& Allen, 2014) reveals only five structures of imidazole derivatives with a $\mathrm{CH}_{2} \mathrm{COOCH}_{2} \mathrm{CH}_{3}$ substituent on nitrogen (Cai et al., 2014; Bahnous et al., 2013; Zaprutko et al., 2012). Imidazoles with benzoyl substituents are slightly more common with eight occurrences (Xue et al., 2014; Nagaraj et al., 2012; Samanta et al., 2013), while the structures of only six $p$-tolyl-substituted imidazoles are found (Bu et al., 1996; Fridman et al., 2006, 2009). These searches also reveal the unique nature of the molecule reported here.

\section{Synthesis and crystallization}

The title compound was synthesized from a mixture of 2-(4methoxyphenyl)-2-oxoacetaldehyde $(1 \mathrm{mmol})$, glycine methyl ester hydrochloride $(1 \mathrm{mmol})$ and selenium dioxide $(1 \mathrm{mmol})$ in a basic environment in acetonitrile at $373 \mathrm{~K}$. Crystals suitable for X-ray investigation were obtained by solvent evaporation from the resulting solution in $33 \%$ yield.

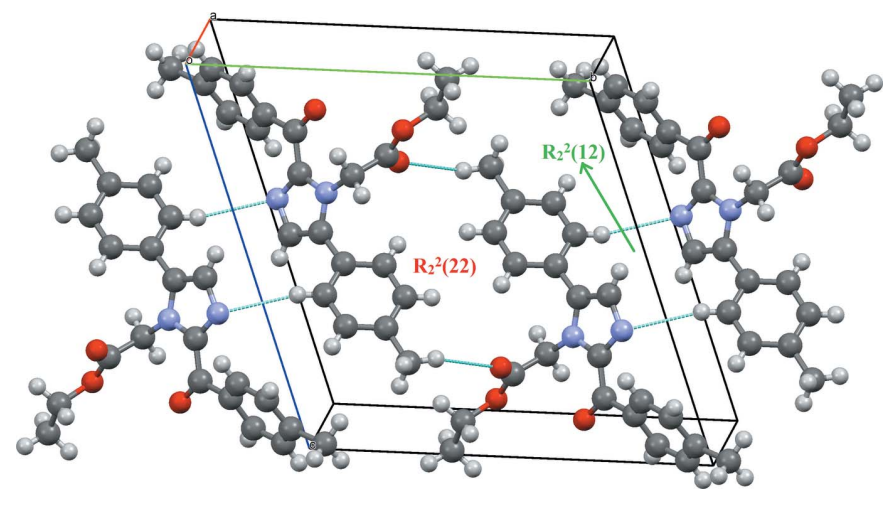

Figure 3

Inversion dimers with $R_{2}^{2}(12)$ and $R_{2}^{2}(22)$ ring motifs resulting from C$\mathrm{H} \cdots \mathrm{N}$ and $\mathrm{C}-\mathrm{H} \cdots \mathrm{O}$ hydrogen bonds. 


\section{Refinement}

Crystal data, data collection and structure refinement details are summarized in Table 2. All $\mathrm{H}$ atoms were positioned geometrically and refined using a riding model, with $\mathrm{C}-\mathrm{H}=$ $0.93-0.97 \AA$ and $U_{\text {iso }}(\mathrm{H})=1.2-1.5 U_{\text {eq }}$ (parent $\mathrm{C}$ atom). The methyl group $\mathrm{C} 22$ of the side chain is disordered over two positions, each with a site-occupancy factor of 0.5 . The atomic displacement parameters of these two $\mathrm{C}$ atoms are restrained to be equivalent and the $\mathrm{C} 21-\mathrm{C} 22$ and $\mathrm{C} 21-\mathrm{C} 22^{\prime}$ bond distances were restrained during the refinement using DFIX commands.

\section{Acknowledgements}

SA and SSK thank the Department of Science and Technology, New Delhi, for financial support of this work through the Fasttrack Young Scientist scheme.

\section{References}

Achar, K. C. S., Hosamani, K. M. \& Seetharamareddy, H. R. (2010). Eur. J. Med. Chem. 45, 2048-2054.

Bahnous, M., Bouraiou, A., Chelghoum, M., Bouacida, S., Roisnel, T., Smati, F., Bentchouala, C., Gros, P. C. \& Belfaitah, A. (2013). Bioorg. Med. Chem. Lett. 23, 1274-1278.

Bernstein, J., Davis, R. E., Shimoni, L. \& Chang, N.-L. (1995). Angew. Chem. Int. Ed. Engl. 34, 1555-1573.

Bhandari, K., Srinivas, N., Keshava, G. B. S. \& Shukla, P. K. (2009). Eur. J. Med. Chem. 44, 437-447.

Bruker (2001). SAINT and SMART. Bruker AXS Inc., Madison, Wisconsin, USA.

Bu, X. R., Li, H., Van Derveer, D. \& Mintz, E. A. (1996). Tetrahedron Lett. 37, 7331-7334.

Cai, Z.-Q., Ma, W.-Y., Liu, J., Hu, Z.-Q., Hou, L. \& Jian-Ya Wang, J.-Y. (2014). Jiegou Huaxue (Chin. J. Struct. Chem.), 33, 1383-1387.

Fridman, N., Kaftory, M., Eichen, Y. \& Speiser, S. (2009). J. Mol. Struct. 917, 101-109.

Fridman, N., Speiser, S. \& Kaftory, M. (2006). Cryst. Growth Des. 6, 2281-2288.

Groom, C. R. \& Allen, F. H. (2014). Angew. Chem. Int. Ed. 53, 662671.

Hadizadeh, F., Hosseinzadeh, H., Sadat Motamed-Shariaty, V., Seifi, M. \& Kazemi, S. (2008). Iranian J. Pharm. Res. 7, 29-33.

Nagaraj, M., Boominathan, M., Muthusubramanian, S. \& Bhuvanesh, N. (2012). Synlett, 23, 1353-1357.

Ozkay, Y., Iskar, I., Incesu, Z. \& Akalin, G. E. (2010). Eur. J. Med. Chem. 45, 1-9.
Table 2

Experimental details.

\begin{tabular}{|c|c|}
\hline \multicolumn{2}{|l|}{ Crystal data } \\
\hline Chemical formula & $\mathrm{C}_{22} \mathrm{H}_{22} \mathrm{~N}_{2} \mathrm{O}_{3}$ \\
\hline$M_{\mathrm{r}}$ & 362.41 \\
\hline Crystal system, space group & Triclinic, $P \overline{1}$ \\
\hline Temperature $(\mathrm{K})$ & 293 \\
\hline$a, b, c(\AA)$ & $\begin{array}{l}5.0968(5), 13.8189(15) \\
\quad 14.6993(17)\end{array}$ \\
\hline$\alpha, \beta, \gamma\left({ }^{\circ}\right)$ & $71.484(5), 84.018(5), 82.531(5)$ \\
\hline$V\left(\AA^{3}\right)$ & $971.20(18)$ \\
\hline$Z$ & 2 \\
\hline Radiation type & Mo $K \alpha$ \\
\hline$\mu\left(\mathrm{mm}^{-1}\right)$ & 0.08 \\
\hline Crystal size $(\mathrm{mm})$ & $0.21 \times 0.19 \times 0.16$ \\
\hline \multicolumn{2}{|l|}{ Data collection } \\
\hline Diffractometer & $\begin{array}{l}\text { Bruker SMART APEX CCD area- } \\
\text { detector }\end{array}$ \\
\hline $\begin{array}{l}\text { No. of measured, independent and } \\
\text { observed }[I>2 \sigma(I)] \text { reflections }\end{array}$ & $18453,3405,2354$ \\
\hline$R_{\text {int }}$ & 0.055 \\
\hline$(\sin \theta / \lambda)_{\max }\left(\AA^{-1}\right)$ & 0.595 \\
\hline \multicolumn{2}{|l|}{ Refinement } \\
\hline$R\left[F^{2}>2 \sigma\left(F^{2}\right)\right], w R\left(F^{2}\right), S$ & $0.054,0.168,1.07$ \\
\hline No. of reflections & 3405 \\
\hline No. of parameters & 251 \\
\hline No. of restraints & 2 \\
\hline $\mathrm{H}$-atom treatment & H-atom parameters constrained \\
\hline$\Delta \rho_{\max }, \Delta \rho_{\min }\left(\mathrm{e} \AA^{-3}\right)$ & $0.52,-0.31$ \\
\hline
\end{tabular}

Computer programs: SMART (Bruker, 2001), SAINT (Bruker, 2001), SHELXTL/PC (Sheldrick, 2008) and PLATON (Spek, 2009).

Pandey, J., Tiwari, V. K., Verma, S. S., Chaturvedi, V., Bhatnagar, S., Sinha, S., Gaikwad, A. N. \& Tripathi, R. P. (2009). Eur. J. Med. Chem. 44, 3350-3355.

Puratchikody, A. \& Doble, M. (2007). Bioorg. Med. Chem. 15, $1083-$ 1090.

Samanta, R. C., De Sarkar, S., Fröhlich, R., Grimme, S. \& Studer, A. (2013). Chem. Sci. 4, 2177-2184.

Sharma, D., Narasimhan, B., Kumar, P., Judge, V., Narang, R., De Clercq, E. \& Balzarini, J. (2009). Eur. J. Med. Chem. 44, 2347-2353.

Sheldrick, G. M. (2008). Acta Cryst. A64, 112-122.

Shingalapur, R. V., Hosamani, K. M. \& Keri, R. S. (2009). Eur. J. Med. Chem. 44, 4244-4248.

Spek, A. L. (2009). Acta Cryst. D65, 148-155.

Xue, W.-J., Li, H.-Z., Gao, F.-F. \& Wu, A. (2014). Tetrahedron, 70, 239-244.

Zaprutko, L., Żwawiak, J., Olender, D. \& Gzella, A. (2012). Heterocycles, 85, 2197-2211. 


\section{supporting information}

Acta Cryst. (2016). E72, 347-349 [doi:10.1107/S2056989016002504]

Crystal structure of ethyl 2-[2-(4-methylbenzoyl)-5-p-tolyl-1 H-imidazol-1yl]acetate

\section{E. Arockia Jeya Yasmi Prabha, S. Suresh Kumar, Anil K. Padala, Qazi Naveed Ahmed and S.}

\section{Athimoolam}

Computing details

Data collection: SMART (Bruker, 2001); cell refinement: SAINT (Bruker, 2001); data reduction: SAINT (Bruker, 2001); program(s) used to solve structure: SHELXTL/PC (Sheldrick, 2008); program(s) used to refine structure: SHELXTL/PC (Sheldrick, 2008); molecular graphics: PLATON (Spek, 2009); software used to prepare material for publication:

SHELXTL/PC (Sheldrick, 2008).

Ethyl 2-[2-(4-methylbenzoyl)-5-p-tolyl-1H-imidazol-1-yl]acetate

\section{Crystal data}

$\mathrm{C}_{22} \mathrm{H}_{22} \mathrm{~N}_{2} \mathrm{O}_{3}$

$M_{r}=362.41$

Triclinic, $P \overline{1}$

$a=5.0968(5) \AA$

$b=13.8189(15) \AA$

$c=14.6993(17) \AA$

$\alpha=71.484(5)^{\circ}$

$\beta=84.018(5)^{\circ}$

$\gamma=82.531(5)^{\circ}$

$V=971.20(18) \AA^{3}$

\section{Data collection}

Bruker SMART APEX CCD area-detector diffractometer

Radiation source: fine-focus sealed tube $\omega$ scans

18453 measured reflections

3405 independent reflections

Refinement

Refinement on $F^{2}$

Least-squares matrix: full

$R\left[F^{2}>2 \sigma\left(F^{2}\right)\right]=0.054$

$w R\left(F^{2}\right)=0.168$

$S=1.07$

3405 reflections

251 parameters

2 restraints
$Z=2$

$F(000)=384$

$D_{\mathrm{x}}=1.239 \mathrm{Mg} \mathrm{m}^{-3}$

Mo $K \alpha$ radiation, $\lambda=0.71073 \AA$

Cell parameters from 2986 reflections

$\theta=2.1-24.4^{\circ}$

$\mu=0.08 \mathrm{~mm}^{-1}$

$T=293 \mathrm{~K}$

Block, colourless

$0.21 \times 0.19 \times 0.16 \mathrm{~mm}$

2354 reflections with $I>2 \sigma(I)$

$R_{\text {int }}=0.055$

$\theta_{\text {max }}=25.0^{\circ}, \theta_{\text {min }}=2.5^{\circ}$

$h=-6 \rightarrow 6$

$k=-16 \rightarrow 16$

$l=-17 \rightarrow 17$

Hydrogen site location: inferred from neighbouring sites

$\mathrm{H}$-atom parameters constrained

$w=1 /\left[\sigma^{2}\left(F_{\mathrm{o}}^{2}\right)+(0.0636 P)^{2}+0.5608 P\right]$

where $P=\left(F_{\mathrm{o}}{ }^{2}+2 F_{\mathrm{c}}{ }^{2}\right) / 3$

$(\Delta / \sigma)_{\max }=0.002$

$\Delta \rho_{\max }=0.52 \mathrm{e}^{-3}$

$\Delta \rho_{\min }=-0.30$ e $\AA^{-3}$ 


\section{Special details}

Geometry. All e.s.d.'s (except the e.s.d. in the dihedral angle between two 1.s. planes) are estimated using the full covariance matrix. The cell e.s.d.'s are taken into account individually in the estimation of e.s.d.'s in distances, angles and torsion angles; correlations between e.s.d.'s in cell parameters are only used when they are defined by crystal symmetry. An approximate (isotropic) treatment of cell e.s.d.'s is used for estimating e.s.d.'s involving l.s. planes.

Fractional atomic coordinates and isotropic or equivalent isotropic displacement parameters $\left(\hat{A}^{2}\right)$

\begin{tabular}{|c|c|c|c|c|c|}
\hline & $x$ & $y$ & $z$ & $U_{\text {iso }} * / U_{\text {eq }}$ & Occ. $(<1)$ \\
\hline $\mathrm{C} 1$ & $-0.6633(7)$ & $0.3819(3)$ & $0.6826(2)$ & $0.0754(9)$ & \\
\hline H1A & -0.6105 & 0.4476 & 0.6779 & $0.113^{*}$ & \\
\hline H1B & -0.6498 & 0.3372 & 0.7474 & $0.113^{*}$ & \\
\hline $\mathrm{H} 1 \mathrm{C}$ & -0.8433 & 0.3901 & 0.6655 & $0.113^{*}$ & \\
\hline $\mathrm{C} 2$ & $-0.4852(5)$ & $0.3362(2)$ & 0.61545 (19) & $0.0530(7)$ & \\
\hline $\mathrm{C} 3$ & -0.5093 & $0.2386(2)$ & $0.6123(2)$ & $0.0568(7)$ & \\
\hline $\mathrm{H} 3$ & -0.6403 & 0.2015 & 0.6517 & $0.068^{*}$ & \\
\hline $\mathrm{C} 4$ & $-0.3441(5)$ & 0.19537 (19) & $0.55220(19)$ & $0.0501(6)$ & \\
\hline $\mathrm{H} 4$ & -0.3636 & 0.1293 & 0.5524 & $0.060^{*}$ & \\
\hline $\mathrm{C} 5$ & $-0.1486(5)$ & $0.24912(17)$ & $0.49132(17)$ & $0.0426(6)$ & \\
\hline C6 & $-0.1244(5)$ & $0.34725(19)$ & $0.4938(2)$ & $0.0536(7)$ & \\
\hline H6 & 0.0048 & 0.3851 & 0.4539 & $0.064 *$ & \\
\hline $\mathrm{C} 7$ & $-0.2906(5)$ & $0.3891(2)$ & $0.5551(2)$ & $0.0573(7)$ & \\
\hline $\mathrm{H} 7$ & -0.2705 & 0.4548 & 0.5557 & $0.069^{*}$ & \\
\hline $\mathrm{C} 8$ & $0.0360(5)$ & $0.19884(17)$ & $0.43313(17)$ & $0.0419(6)$ & \\
\hline C9 & $0.1875(5)$ & $0.10614(18)$ & 0.46091 (18) & $0.0466(6)$ & \\
\hline H9 & 0.1835 & 0.0613 & 0.5233 & $0.056^{*}$ & \\
\hline $\mathrm{C} 10$ & $0.2885(5)$ & $0.16918(17)$ & $0.31157(17)$ & $0.0428(6)$ & \\
\hline $\mathrm{C} 11$ & $0.4033(5)$ & $0.1806(2)$ & 0.21394 (19) & 0.0539 (7) & \\
\hline $\mathrm{C} 12$ & $0.6016(5)$ & $0.0980(2)$ & $0.19780(17)$ & $0.0480(6)$ & \\
\hline $\mathrm{C} 17$ & $0.8022(5)$ & $0.0507(2)$ & $0.25858(19)$ & $0.0514(7)$ & \\
\hline H17 & 0.8186 & 0.0717 & 0.3117 & $0.062 *$ & \\
\hline $\mathrm{C} 16$ & $0.9772(6)$ & $-0.0268(2)$ & $0.2414(2)$ & $0.0614(8)$ & \\
\hline H16 & 1.1136 & -0.0560 & 0.2821 & $0.074 *$ & \\
\hline $\mathrm{C} 15$ & $0.9556(6)$ & $-0.0622(2)$ & $0.1653(2)$ & $0.0629(8)$ & \\
\hline $\mathrm{C} 14$ & $0.7592(7)$ & $-0.0130(3)$ & $0.1034(2)$ & $0.0777(10)$ & \\
\hline H14 & 0.7426 & -0.0345 & 0.0505 & $0.093 *$ & \\
\hline $\mathrm{C} 13$ & $0.5874(6)$ & $0.0671(3)$ & $0.1182(2)$ & $0.0709(9)$ & \\
\hline H13 & 0.4612 & 0.1004 & 0.0742 & $0.085^{*}$ & \\
\hline C18 & $1.1384(8)$ & $-0.1519(3)$ & $0.1513(3)$ & $0.0990(13)$ & \\
\hline H18A & 1.0844 & -0.1703 & 0.0988 & $0.149^{*}$ & \\
\hline H18B & 1.1306 & -0.2092 & 0.2089 & $0.149^{*}$ & \\
\hline $\mathrm{H} 18 \mathrm{C}$ & 1.3167 & -0.1334 & 0.1372 & $0.149 *$ & \\
\hline C19 & $-0.0310(5)$ & $0.33098(18)$ & $0.27136(18)$ & $0.0488(6)$ & \\
\hline H19A & -0.1902 & 0.3538 & 0.3044 & $0.059 *$ & \\
\hline H19B & -0.0836 & 0.3150 & 0.2167 & $0.059^{*}$ & \\
\hline C20 & $0.1464(5)$ & $0.41606(19)$ & $0.23635(19)$ & $0.0510(7)$ & \\
\hline $\mathrm{C} 21$ & $0.2175(8)$ & $0.5710(2)$ & $0.1156(2)$ & $0.0923(12)$ & \\
\hline $\mathrm{H} 21 \mathrm{~A}$ & 0.4038 & 0.5456 & 0.1198 & $0.111 *$ & \\
\hline
\end{tabular}




$\begin{array}{llllll}\text { H21B } & 0.1794 & 0.6196 & 0.1517 & 0.111^{*} & \\ \text { C22 } & 0.161(3) & 0.6255(15) & 0.0101(4) & 0.119(4) & 0.5 \\ \text { H22A } & 0.2473 & 0.5850 & -0.0289 & 0.179 * & 0.5 \\ \text { H22B } & 0.2273 & 0.6915 & -0.0105 & 0.179 * & 0.5 \\ \text { H22C } & -0.0267 & 0.6340 & 0.0036 & 0.179 * & 0.5 \\ \text { C22' } & 0.047(3) & 0.6433(15) & 0.0364(5) & 0.119(4) & 0.5 \\ \text { H22D } & 0.0075 & 0.6068 & -0.0055 & 0.179 * & 0.5 \\ \text { H22E } & 0.1418 & 0.7005 & 0.0001 & 0.179 * & 0.5 \\ \text { H22F } & -0.1156 & 0.6677 & 0.0651 & 0.0464(5) & 0.5 \\ \text { N1 } & 0.3431(4) & 0.08765(14) & 0.38698(14) & 0.0434(5) & 0.0913(8) \\ \text { N2 } & 0.1006(4) & 0.23871(14) & 0.33651(14) & 0.0774(7) & 0.0663(6) \\ \text { O1 } & 0.3314(5) & 0.25504(18) & 0.14648(15) & 0.27496(17) & \\ \text { O2 } & 0.3358(4) & 0.42182(16) & 0.15783(13) & & \\ \text { O3 } & 0.0563(4) & 0.48554(13) & & & \end{array}$

Atomic displacement parameters $\left(\AA^{2}\right)$

\begin{tabular}{|c|c|c|c|c|c|c|}
\hline & $U^{11}$ & $U^{22}$ & $U^{33}$ & $U^{12}$ & $U^{13}$ & $U^{23}$ \\
\hline $\mathrm{C} 1$ & $0.077(2)$ & $0.081(2)$ & 0.077 (2) & $0.0039(17)$ & $-0.0022(17)$ & $-0.0423(18)$ \\
\hline $\mathrm{C} 2$ & $0.0517(15)$ & $0.0546(16)$ & $0.0558(16)$ & $0.0006(13)$ & -0.0118 & $-0.0211(13)$ \\
\hline $\mathrm{C} 3$ & $0.0558(16)$ & $0.0555(17)$ & $0.0601(17)$ & $-0.0134(13)$ & $0.0026(13)$ & $-0.0185(14)$ \\
\hline $\mathrm{C} 4$ & $0.0553(15)$ & 0.0386 (13) & 0.0585 (16) & $-0.0095(12)$ & $-0.0050(13)$ & $-0.0155(12)$ \\
\hline $\mathrm{C} 5$ & $0.0453(13)$ & $0.0361(12)$ & $0.0458(14)$ & $-0.0031(10)$ & $-0.0120(11)$ & $-0.0094(11)$ \\
\hline C6 & $0.0531(15)$ & 0.0392 (14) & $0.0681(18)$ & $-0.0111(12)$ & $-0.0003(13)$ & $-0.0149(13)$ \\
\hline $\mathrm{C} 7$ & 0.0617 (17) & 0.0408 (14) & 0.0759 (19) & $-0.0036(13)$ & $-0.0105(15)$ & $-0.0259(14)$ \\
\hline $\mathrm{C} 8$ & $0.0475(14)$ & $0.0331(12)$ & $0.0450(14)$ & $-0.0075(10)$ & $-0.0073(11)$ & $-0.0092(11)$ \\
\hline $\mathrm{C} 9$ & $0.0595(16)$ & $0.0362(13)$ & 0.0405 (14) & $-0.0052(11)$ & $-0.0059(12)$ & $-0.0060(11)$ \\
\hline $\mathrm{C} 10$ & $0.0459(14)$ & $0.0346(12)$ & $0.0447(14)$ & $-0.0027(10)$ & $-0.0072(11)$ & $-0.0071(11)$ \\
\hline C11 & $0.0557(16)$ & $0.0496(15)$ & $0.0467(15)$ & $-0.0009(12)$ & $-0.0049(12)$ & $-0.0025(12)$ \\
\hline $\mathrm{C} 12$ & $0.0499(15)$ & $0.0515(15)$ & $0.0401(14)$ & $-0.0078(12)$ & $-0.0016(11)$ & $-0.0099(11)$ \\
\hline $\mathrm{C} 17$ & $0.0535(15)$ & $0.0524(15)$ & $0.0478(15)$ & $-0.0058(13)$ & $-0.0060(12)$ & $-0.0136(12)$ \\
\hline $\mathrm{C} 16$ & $0.0600(17)$ & $0.0582(17)$ & $0.0561(17)$ & $0.0031(14)$ & $-0.0026(13)$ & $-0.0077(14)$ \\
\hline $\mathrm{C} 15$ & 0.0662 (19) & $0.0526(17)$ & 0.0669 (19) & $-0.0124(14)$ & $0.0146(15)$ & $-0.0175(15)$ \\
\hline $\mathrm{C} 14$ & $0.074(2)$ & 0.109 (3) & $0.070(2)$ & $-0.016(2)$ & $0.0051(17)$ & $-0.055(2)$ \\
\hline C13 & $0.0578(18)$ & $0.106(3)$ & $0.0529(17)$ & $0.0029(17)$ & $-0.0105(14)$ & $-0.0325(17)$ \\
\hline C18 & $0.116(3)$ & $0.063(2)$ & $0.112(3)$ & $-0.003(2)$ & $0.032(2)$ & $-0.032(2)$ \\
\hline C19 & $0.0471(14)$ & $0.0397(13)$ & $0.0500(15)$ & $0.0019(11)$ & $-0.0091(11)$ & -0.0010 \\
\hline $\mathrm{C} 20$ & $0.0540(16)$ & $0.0381(14)$ & $0.0522(15)$ & $0.0030(12)$ & $-0.0057(13)$ & $-0.0041(12)$ \\
\hline $\mathrm{C} 21$ & $0.133(3)$ & $0.0462(18)$ & $0.085(2)$ & $-0.0256(19)$ & $-0.012(2)$ & $0.0059(17)$ \\
\hline $\mathrm{C} 22$ & $0.199(13)$ & $0.099(7)$ & $0.050(4)$ & $-0.063(7)$ & $0.004(6)$ & $0.004(6)$ \\
\hline $\mathrm{C} 22^{\prime}$ & 0.199 (13) & $0.099(7)$ & $0.050(4)$ & $-0.063(7)$ & $0.004(6)$ & $0.004(6)$ \\
\hline N1 & $0.0566(13)$ & $0.0345(11)$ & $0.0443(12)$ & $-0.0018(9)$ & $-0.0086(10)$ & $-0.0061(9)$ \\
\hline N2 & $0.0462(11)$ & $0.0331(10)$ & $0.0448(12)$ & $-0.0018(9)$ & $-0.0081(9)$ & $-0.0029(9)$ \\
\hline O1 & $0.1031(18)$ & $0.0828(16)$ & $0.0517(12)$ & $0.0282(14)$ & $0.0041(12)$ & $0.0128(11)$ \\
\hline $\mathrm{O} 2$ & 0.0675 (13) & $0.0612(13)$ & $0.0934(16)$ & $-0.0175(11)$ & $-0.0261(12)$ & $0.0010(11)$ \\
\hline $\mathrm{O} 3$ & $0.0886(14)$ & $0.0412(10)$ & 0.0577 (12) & $-0.0074(10)$ & $-0.0135(10)$ & 0.0037 (9) \\
\hline
\end{tabular}


Geometric parameters $\left(\AA,{ }^{\circ}\right)$

\begin{tabular}{|c|c|c|c|}
\hline $\mathrm{C} 1-\mathrm{C} 2$ & $1.503(4)$ & $\mathrm{C} 16-\mathrm{C} 15$ & $1.375(4)$ \\
\hline $\mathrm{C} 1-\mathrm{H} 1 \mathrm{~A}$ & 0.9600 & C16-H16 & 0.9300 \\
\hline $\mathrm{C} 1-\mathrm{H} 1 \mathrm{~B}$ & 0.9600 & $\mathrm{C} 15-\mathrm{C} 14$ & $1.383(4)$ \\
\hline $\mathrm{C} 1-\mathrm{H} 1 \mathrm{C}$ & 0.9600 & $\mathrm{C} 15-\mathrm{C} 18$ & $1.506(4)$ \\
\hline $\mathrm{C} 2-\mathrm{C} 7$ & $1.377(4)$ & $\mathrm{C} 14-\mathrm{C} 13$ & $1.379(4)$ \\
\hline $\mathrm{C} 2-\mathrm{C} 3$ & $1.386(4)$ & C14-H14 & 0.9300 \\
\hline $\mathrm{C} 3-\mathrm{C} 4$ & $1.377(4)$ & $\mathrm{C} 13-\mathrm{H} 13$ & 0.9300 \\
\hline $\mathrm{C} 3-\mathrm{H} 3$ & 0.9300 & $\mathrm{C} 18-\mathrm{H} 18 \mathrm{~A}$ & 0.9600 \\
\hline $\mathrm{C} 4-\mathrm{C} 5$ & $1.389(3)$ & C18-H18B & 0.9600 \\
\hline $\mathrm{C} 4-\mathrm{H} 4$ & 0.9300 & $\mathrm{C} 18-\mathrm{H} 18 \mathrm{C}$ & 0.9600 \\
\hline $\mathrm{C} 5-\mathrm{C} 6$ & $1.390(3)$ & $\mathrm{C} 19-\mathrm{N} 2$ & $1.459(3)$ \\
\hline $\mathrm{C} 5-\mathrm{C} 8$ & $1.463(3)$ & $\mathrm{C} 19-\mathrm{C} 20$ & $1.503(4)$ \\
\hline $\mathrm{C} 6-\mathrm{C} 7$ & $1.381(4)$ & C19-H19A & 0.9700 \\
\hline $\mathrm{C} 6-\mathrm{H} 6$ & 0.9300 & C19-H19B & 0.9700 \\
\hline $\mathrm{C} 7-\mathrm{H} 7$ & 0.9300 & $\mathrm{C} 20-\mathrm{O} 2$ & $1.193(3)$ \\
\hline $\mathrm{C} 8-\mathrm{C} 9$ & $1.371(3)$ & $\mathrm{C} 20-\mathrm{O} 3$ & $1.325(3)$ \\
\hline $\mathrm{C} 8-\mathrm{N} 2$ & $1.372(3)$ & $\mathrm{C} 21-\mathrm{O} 3$ & $1.462(4)$ \\
\hline $\mathrm{C} 9-\mathrm{N} 1$ & $1.349(3)$ & $\mathrm{C} 21-\mathrm{C} 22$ & $1.534(2)$ \\
\hline C9- $\mathrm{H} 9$ & 0.9300 & $\mathrm{C} 21-\mathrm{C} 22^{\prime}$ & $1.535(2)$ \\
\hline $\mathrm{C} 10-\mathrm{N} 1$ & $1.329(3)$ & $\mathrm{C} 21-\mathrm{H} 21 \mathrm{~A}$ & 0.9700 \\
\hline $\mathrm{C} 10-\mathrm{N} 2$ & $1.373(3)$ & $\mathrm{C} 21-\mathrm{H} 21 \mathrm{~B}$ & 0.9700 \\
\hline $\mathrm{C} 10-\mathrm{C} 11$ & $1.460(4)$ & $\mathrm{C} 22-\mathrm{H} 22 \mathrm{~A}$ & 0.9600 \\
\hline $\mathrm{C} 11-\mathrm{O} 1$ & $1.227(3)$ & $\mathrm{C} 22-\mathrm{H} 22 \mathrm{~B}$ & 0.9600 \\
\hline $\mathrm{C} 11-\mathrm{C} 12$ & $1.484(4)$ & $\mathrm{C} 22-\mathrm{H} 22 \mathrm{C}$ & 0.9600 \\
\hline $\mathrm{C} 12-\mathrm{C} 13$ & $1.379(4)$ & $\mathrm{C} 22^{\prime}-\mathrm{H} 22 \mathrm{D}$ & 0.9600 \\
\hline $\mathrm{C} 12-\mathrm{C} 17$ & $1.384(3)$ & $\mathrm{C} 22^{\prime}-\mathrm{H} 22 \mathrm{E}$ & 0.9600 \\
\hline $\mathrm{C} 17-\mathrm{C} 16$ & $1.373(4)$ & $\mathrm{C} 22^{\prime}-\mathrm{H} 22 \mathrm{~F}$ & 0.9600 \\
\hline C17-H17 & 0.9300 & & \\
\hline $\mathrm{C} 2-\mathrm{C} 1-\mathrm{H} 1 \mathrm{~A}$ & 109.5 & $\mathrm{C} 14-\mathrm{C} 15-\mathrm{C} 18$ & $121.8(3)$ \\
\hline $\mathrm{C} 2-\mathrm{C} 1-\mathrm{H} 1 \mathrm{~B}$ & 109.5 & $\mathrm{C} 13-\mathrm{C} 14-\mathrm{C} 15$ & $121.5(3)$ \\
\hline $\mathrm{H} 1 \mathrm{~A}-\mathrm{C} 1-\mathrm{H} 1 \mathrm{~B}$ & 109.5 & $\mathrm{C} 13-\mathrm{C} 14-\mathrm{H} 14$ & 119.2 \\
\hline $\mathrm{C} 2-\mathrm{C} 1-\mathrm{H} 1 \mathrm{C}$ & 109.5 & $\mathrm{C} 15-\mathrm{C} 14-\mathrm{H} 14$ & 119.2 \\
\hline $\mathrm{H} 1 \mathrm{~A}-\mathrm{C} 1-\mathrm{H} 1 \mathrm{C}$ & 109.5 & $\mathrm{C} 12-\mathrm{C} 13-\mathrm{C} 14$ & $120.4(3)$ \\
\hline $\mathrm{H} 1 \mathrm{~B}-\mathrm{C} 1-\mathrm{H} 1 \mathrm{C}$ & 109.5 & $\mathrm{C} 12-\mathrm{C} 13-\mathrm{H} 13$ & 119.8 \\
\hline $\mathrm{C} 7-\mathrm{C} 2-\mathrm{C} 3$ & $117.3(3)$ & $\mathrm{C} 14-\mathrm{C} 13-\mathrm{H} 13$ & 119.8 \\
\hline $\mathrm{C} 7-\mathrm{C} 2-\mathrm{C} 1$ & $121.5(3)$ & $\mathrm{C} 15-\mathrm{C} 18-\mathrm{H} 18 \mathrm{~A}$ & 109.5 \\
\hline $\mathrm{C} 3-\mathrm{C} 2-\mathrm{C} 1$ & $121.2(3)$ & $\mathrm{C} 15-\mathrm{C} 18-\mathrm{H} 18 \mathrm{~B}$ & 109.5 \\
\hline $\mathrm{C} 4-\mathrm{C} 3-\mathrm{C} 2$ & $121.6(3)$ & $\mathrm{H} 18 \mathrm{~A}-\mathrm{C} 18-\mathrm{H} 18 \mathrm{~B}$ & 109.5 \\
\hline $\mathrm{C} 4-\mathrm{C} 3-\mathrm{H} 3$ & 119.2 & $\mathrm{C} 15-\mathrm{C} 18-\mathrm{H} 18 \mathrm{C}$ & 109.5 \\
\hline $\mathrm{C} 2-\mathrm{C} 3-\mathrm{H} 3$ & 119.2 & $\mathrm{H} 18 \mathrm{~A}-\mathrm{C} 18-\mathrm{H} 18 \mathrm{C}$ & 109.5 \\
\hline $\mathrm{C} 3-\mathrm{C} 4-\mathrm{C} 5$ & $120.8(2)$ & $\mathrm{H} 18 \mathrm{~B}-\mathrm{C} 18-\mathrm{H} 18 \mathrm{C}$ & 109.5 \\
\hline $\mathrm{C} 3-\mathrm{C} 4-\mathrm{H} 4$ & 119.6 & $\mathrm{~N} 2-\mathrm{C} 19-\mathrm{C} 20$ & $111.7(2)$ \\
\hline $\mathrm{C} 5-\mathrm{C} 4-\mathrm{H} 4$ & 119.6 & $\mathrm{~N} 2-\mathrm{C} 19-\mathrm{H} 19 \mathrm{~A}$ & 109.3 \\
\hline $\mathrm{C} 4-\mathrm{C} 5-\mathrm{C} 6$ & $117.8(2)$ & $\mathrm{C} 20-\mathrm{C} 19-\mathrm{H} 19 \mathrm{~A}$ & 109.3 \\
\hline $\mathrm{C} 4-\mathrm{C} 5-\mathrm{C} 8$ & $119.7(2)$ & N2-C19-H19B & 109.3 \\
\hline
\end{tabular}




\begin{tabular}{|c|c|c|c|}
\hline $\mathrm{C} 6-\mathrm{C} 5-\mathrm{C} 8$ & $122.3(2)$ & $\mathrm{C} 20-\mathrm{C} 19-\mathrm{H} 19 \mathrm{~B}$ & 109.3 \\
\hline $\mathrm{C} 7-\mathrm{C} 6-\mathrm{C} 5$ & $120.5(2)$ & $\mathrm{H} 19 \mathrm{~A}-\mathrm{C} 19-\mathrm{H} 19 \mathrm{~B}$ & 107.9 \\
\hline $\mathrm{C} 7-\mathrm{C} 6-\mathrm{H} 6$ & 119.7 & $\mathrm{O} 2-\mathrm{C} 20-\mathrm{O} 3$ & $124.9(2)$ \\
\hline $\mathrm{C} 5-\mathrm{C} 6-\mathrm{H} 6$ & 119.7 & $\mathrm{O} 2-\mathrm{C} 20-\mathrm{C} 19$ & $125.1(2)$ \\
\hline $\mathrm{C} 2-\mathrm{C} 7-\mathrm{C} 6$ & $121.9(2)$ & $\mathrm{O} 3-\mathrm{C} 20-\mathrm{C} 19$ & $109.9(2)$ \\
\hline $\mathrm{C} 2-\mathrm{C} 7-\mathrm{H} 7$ & 119.1 & $\mathrm{O} 3-\mathrm{C} 21-\mathrm{C} 22$ & $111.1(9)$ \\
\hline $\mathrm{C} 6-\mathrm{C} 7-\mathrm{H} 7$ & 119.1 & $\mathrm{O} 3-\mathrm{C} 21-\mathrm{C} 22^{\prime}$ & $102.4(9)$ \\
\hline $\mathrm{C} 9-\mathrm{C} 8-\mathrm{N} 2$ & $104.8(2)$ & $\mathrm{O} 3-\mathrm{C} 21-\mathrm{H} 21 \mathrm{~A}$ & 109.4 \\
\hline $\mathrm{C} 9-\mathrm{C} 8-\mathrm{C} 5$ & $129.3(2)$ & $\mathrm{C} 22-\mathrm{C} 21-\mathrm{H} 21 \mathrm{~A}$ & 109.4 \\
\hline $\mathrm{N} 2-\mathrm{C} 8-\mathrm{C} 5$ & $125.8(2)$ & $\mathrm{O} 3-\mathrm{C} 21-\mathrm{H} 21 \mathrm{~B}$ & 109.4 \\
\hline $\mathrm{N} 1-\mathrm{C} 9-\mathrm{C} 8$ & $112.0(2)$ & $\mathrm{C} 22-\mathrm{C} 21-\mathrm{H} 21 \mathrm{~B}$ & 109.4 \\
\hline $\mathrm{N} 1-\mathrm{C} 9-\mathrm{H} 9$ & 124.0 & $\mathrm{H} 21 \mathrm{~A}-\mathrm{C} 21-\mathrm{H} 21 \mathrm{~B}$ & 108.0 \\
\hline $\mathrm{C} 8-\mathrm{C} 9-\mathrm{H} 9$ & 124.0 & $\mathrm{C} 21-\mathrm{C} 22-\mathrm{H} 22 \mathrm{~A}$ & 109.5 \\
\hline $\mathrm{N} 1-\mathrm{C} 10-\mathrm{N} 2$ & $111.2(2)$ & $\mathrm{C} 21-\mathrm{C} 22-\mathrm{H} 22 \mathrm{~B}$ & 109.5 \\
\hline $\mathrm{N} 1-\mathrm{C} 10-\mathrm{C} 11$ & $124.2(2)$ & $\mathrm{H} 22 \mathrm{~A}-\mathrm{C} 22-\mathrm{H} 22 \mathrm{~B}$ & 109.5 \\
\hline $\mathrm{N} 2-\mathrm{C} 10-\mathrm{C} 11$ & $124.5(2)$ & $\mathrm{C} 21-\mathrm{C} 22-\mathrm{H} 22 \mathrm{C}$ & 109.5 \\
\hline $\mathrm{O} 1-\mathrm{C} 11-\mathrm{C} 10$ & $120.8(2)$ & $\mathrm{H} 22 \mathrm{~A}-\mathrm{C} 22-\mathrm{H} 22 \mathrm{C}$ & 109.5 \\
\hline $\mathrm{O} 1-\mathrm{C} 11-\mathrm{C} 12$ & $120.8(2)$ & $\mathrm{H} 22 \mathrm{~B}-\mathrm{C} 22-\mathrm{H} 22 \mathrm{C}$ & 109.5 \\
\hline $\mathrm{C} 10-\mathrm{C} 11-\mathrm{C} 12$ & $118.3(2)$ & $\mathrm{C} 21-\mathrm{C} 22^{\prime}-\mathrm{H} 22 \mathrm{D}$ & 109.5 \\
\hline $\mathrm{C} 13-\mathrm{C} 12-\mathrm{C} 17$ & $118.2(3)$ & $\mathrm{C} 21-\mathrm{C} 22^{\prime}-\mathrm{H} 22 \mathrm{E}$ & 109.5 \\
\hline $\mathrm{C} 13-\mathrm{C} 12-\mathrm{C} 11$ & $118.7(2)$ & $\mathrm{H} 22 \mathrm{D}-\mathrm{C} 22^{\prime}-\mathrm{H} 22 \mathrm{E}$ & 109.5 \\
\hline $\mathrm{C} 17-\mathrm{C} 12-\mathrm{C} 11$ & $123.2(2)$ & $\mathrm{C} 21-\mathrm{C} 22^{\prime}-\mathrm{H} 22 \mathrm{~F}$ & 109.5 \\
\hline $\mathrm{C} 16-\mathrm{C} 17-\mathrm{C} 12$ & $120.8(3)$ & $\mathrm{H} 22 \mathrm{D}-\mathrm{C} 22^{\prime}-\mathrm{H} 22 \mathrm{~F}$ & 109.5 \\
\hline $\mathrm{C} 16-\mathrm{C} 17-\mathrm{H} 17$ & 119.6 & $\mathrm{H} 22 \mathrm{E}-\mathrm{C} 22^{\prime}-\mathrm{H} 22 \mathrm{~F}$ & 109.5 \\
\hline $\mathrm{C} 12-\mathrm{C} 17-\mathrm{H} 17$ & 119.6 & $\mathrm{C} 10-\mathrm{N} 1-\mathrm{C} 9$ & $105.0(2)$ \\
\hline $\mathrm{C} 17-\mathrm{C} 16-\mathrm{C} 15$ & $121.5(3)$ & $\mathrm{C} 8-\mathrm{N} 2-\mathrm{C} 10$ & $106.94(18)$ \\
\hline $\mathrm{C} 17-\mathrm{C} 16-\mathrm{H} 16$ & 119.2 & $\mathrm{C} 8-\mathrm{N} 2-\mathrm{C} 19$ & $125.8(2)$ \\
\hline $\mathrm{C} 15-\mathrm{C} 16-\mathrm{H} 16$ & 119.2 & $\mathrm{C} 10-\mathrm{N} 2-\mathrm{C} 19$ & $126.8(2)$ \\
\hline $\mathrm{C} 16-\mathrm{C} 15-\mathrm{C} 14$ & $117.4(3)$ & $\mathrm{C} 20-\mathrm{O} 3-\mathrm{C} 21$ & $114.8(2)$ \\
\hline $\mathrm{C} 16-\mathrm{C} 15-\mathrm{C} 18$ & $120.8(3)$ & & \\
\hline $\mathrm{C} 7-\mathrm{C} 2-\mathrm{C} 3-\mathrm{C} 4$ & $0.7(4)$ & $\mathrm{C} 17-\mathrm{C} 16-\mathrm{C} 15-\mathrm{C} 14$ & $3.4(4)$ \\
\hline $\mathrm{C} 1-\mathrm{C} 2-\mathrm{C} 3-\mathrm{C} 4$ & $-178.8(3)$ & $\mathrm{C} 17-\mathrm{C} 16-\mathrm{C} 15-\mathrm{C} 18$ & $-176.0(3)$ \\
\hline $\mathrm{C} 2-\mathrm{C} 3-\mathrm{C} 4-\mathrm{C} 5$ & $-0.9(4)$ & $\mathrm{C} 16-\mathrm{C} 15-\mathrm{C} 14-\mathrm{C} 13$ & $-1.4(5)$ \\
\hline $\mathrm{C} 3-\mathrm{C} 4-\mathrm{C} 5-\mathrm{C} 6$ & $0.6(4)$ & $\mathrm{C} 18-\mathrm{C} 15-\mathrm{C} 14-\mathrm{C} 13$ & $178.0(3)$ \\
\hline $\mathrm{C} 3-\mathrm{C} 4-\mathrm{C} 5-\mathrm{C} 8$ & $175.8(2)$ & $\mathrm{C} 17-\mathrm{C} 12-\mathrm{C} 13-\mathrm{C} 14$ & $3.8(4)$ \\
\hline $\mathrm{C} 4-\mathrm{C} 5-\mathrm{C} 6-\mathrm{C} 7$ & $0.0(4)$ & $\mathrm{C} 11-\mathrm{C} 12-\mathrm{C} 13-\mathrm{C} 14$ & $-176.6(3)$ \\
\hline $\mathrm{C} 8-\mathrm{C} 5-\mathrm{C} 6-\mathrm{C} 7$ & $-175.1(2)$ & $\mathrm{C} 15-\mathrm{C} 14-\mathrm{C} 13-\mathrm{C} 12$ & $-2.3(5)$ \\
\hline $\mathrm{C} 3-\mathrm{C} 2-\mathrm{C} 7-\mathrm{C} 6$ & $-0.2(4)$ & $\mathrm{N} 2-\mathrm{C} 19-\mathrm{C} 20-\mathrm{O} 2$ & $-20.6(4)$ \\
\hline $\mathrm{C} 1-\mathrm{C} 2-\mathrm{C} 7-\mathrm{C} 6$ & $179.4(3)$ & $\mathrm{N} 2-\mathrm{C} 19-\mathrm{C} 20-\mathrm{O} 3$ & $161.4(2)$ \\
\hline $\mathrm{C} 5-\mathrm{C} 6-\mathrm{C} 7-\mathrm{C} 2$ & $-0.2(4)$ & $\mathrm{N} 2-\mathrm{C} 10-\mathrm{N} 1-\mathrm{C} 9$ & $-0.2(3)$ \\
\hline $\mathrm{C} 4-\mathrm{C} 5-\mathrm{C} 8-\mathrm{C} 9$ & $-51.0(4)$ & $\mathrm{C} 11-\mathrm{C} 10-\mathrm{N} 1-\mathrm{C} 9$ & $-177.1(2)$ \\
\hline $\mathrm{C} 6-\mathrm{C} 5-\mathrm{C} 8-\mathrm{C} 9$ & $124.0(3)$ & $\mathrm{C} 8-\mathrm{C} 9-\mathrm{N} 1-\mathrm{C} 10$ & $-0.3(3)$ \\
\hline $\mathrm{C} 4-\mathrm{C} 5-\mathrm{C} 8-\mathrm{N} 2$ & 131.5 & $\mathrm{C} 9-\mathrm{C} 8-\mathrm{N} 2-\mathrm{C} 10$ & $-0.7(3)$ \\
\hline $\mathrm{C} 6-\mathrm{C} 5-\mathrm{C} 8-\mathrm{N} 2$ & $-53.5(4)$ & $\mathrm{C} 5-\mathrm{C} 8-\mathrm{N} 2-\mathrm{C} 10$ & $177.3(2)$ \\
\hline $\mathrm{N} 2-\mathrm{C} 8-\mathrm{C} 9-\mathrm{N} 1$ & $0.7(3)$ & $\mathrm{C} 9-\mathrm{C} 8-\mathrm{N} 2-\mathrm{C} 19$ & $172.2(2)$ \\
\hline $\mathrm{C} 5-\mathrm{C} 8-\mathrm{C} 9-\mathrm{N} 1$ & $-177.2(2)$ & $\mathrm{C} 5-\mathrm{C} 8-\mathrm{N} 2-\mathrm{C} 19$ & $-9.8(4)$ \\
\hline $\mathrm{N} 1-\mathrm{C} 10-\mathrm{C} 11-\mathrm{O} 1$ & $175.3(3)$ & $\mathrm{N} 1-\mathrm{C} 10-\mathrm{N} 2-\mathrm{C} 8$ & $0.6(3)$ \\
\hline
\end{tabular}




$\begin{array}{ll}\mathrm{N} 2-\mathrm{C} 10-\mathrm{C} 11-\mathrm{O} 1 & -1.2(4) \\ \mathrm{N} 1-\mathrm{C} 10-\mathrm{C} 11-\mathrm{C} 12 & -2.5(4) \\ \mathrm{N} 2-\mathrm{C} 10-\mathrm{C} 11-\mathrm{C} 12 & -179.0(2) \\ \mathrm{O} 1-\mathrm{C} 11-\mathrm{C} 12-\mathrm{C} 13 & -39.9(4) \\ \mathrm{C} 10-\mathrm{C} 11-\mathrm{C} 12-\mathrm{C} 13 & 137.9(3) \\ \mathrm{O} 1-\mathrm{C} 11-\mathrm{C} 12-\mathrm{C} 17 & 139.7(3) \\ \mathrm{C} 10-\mathrm{C} 11-\mathrm{C} 12-\mathrm{C} 17 & -42.6(4) \\ \mathrm{C} 13-\mathrm{C} 12-\mathrm{C} 17-\mathrm{C} 16 & -1.8(4) \\ \mathrm{C} 11-\mathrm{C} 12-\mathrm{C} 17-\mathrm{C} 16 & 178.6(2) \\ \mathrm{C} 12-\mathrm{C} 17-\mathrm{C} 16-\mathrm{C} 15 & -1.9(4)\end{array}$

$177.5(2)$

$-172.3(2)$

4.7 (4)

$111.7(3)$

$-76.8(3)$

$3.5(4)$

$-178.5(2)$

$160.0(6)$

$-172.9(6)$

Hydrogen-bond geometry $\left(\AA,{ }^{\circ}\right)$

\begin{tabular}{lllll}
\hline$D-\mathrm{H} \cdots A$ & $D-\mathrm{H}$ & $\mathrm{H} \cdots A$ & $D \cdots A$ & $D-\mathrm{H} \cdots A$ \\
\hline $\mathrm{C} 6-\mathrm{H} 6 \cdots \mathrm{O} 2$ & 0.93 & 2.91 & $3.723(4)$ & 147 \\
$\mathrm{C} 1-\mathrm{H} 1 A \cdots \mathrm{O} 2^{\mathrm{i}}$ & 0.96 & 2.71 & $3.605(4)$ & 155 \\
$\mathrm{C} 4-\mathrm{H} 4 \cdots \mathrm{N} 1^{\mathrm{ii}}$ & 0.93 & 2.83 & $3.724(3)$ & 161 \\
$\mathrm{C} 19-\mathrm{H} 19 A \cdots \mathrm{O} 2^{\mathrm{iii}}$ & 0.97 & 2.51 & $3.309(3)$ & 140 \\
\hline
\end{tabular}

Symmetry codes: (i) $-x,-y+1,-z+1$; (ii) $-x,-y,-z+1$; (iii) $x-1, y, z$. 